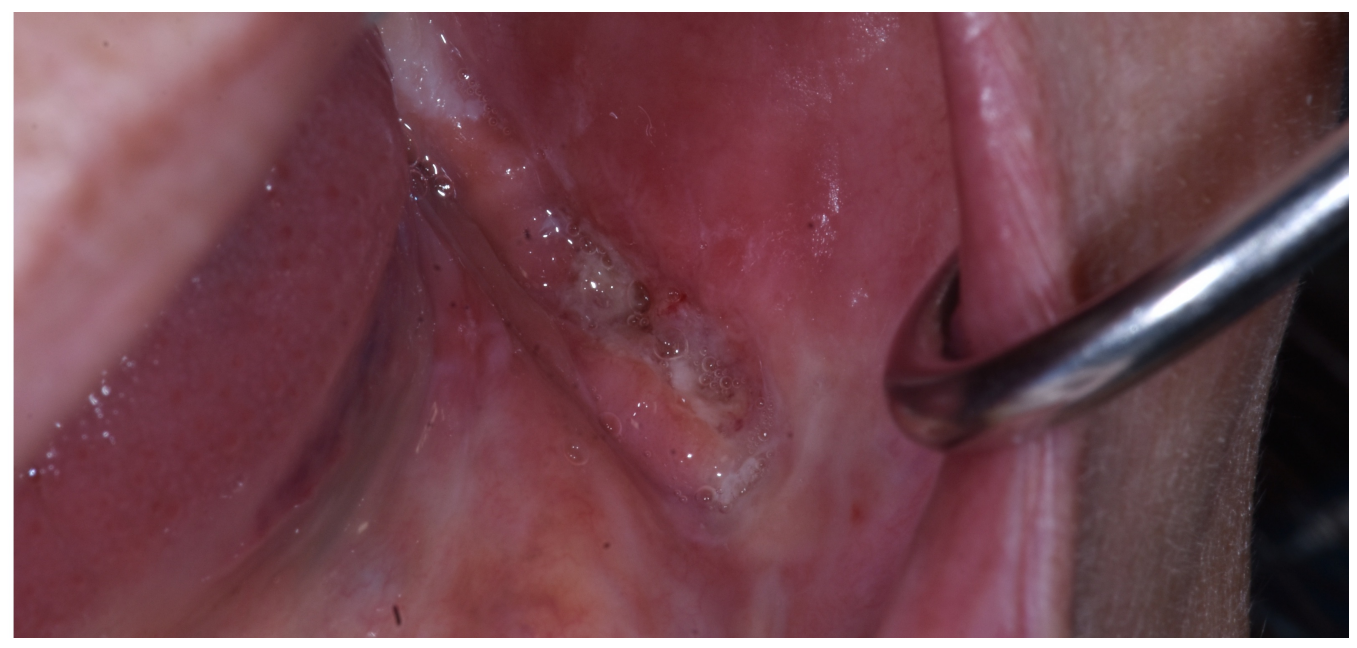

\title{
Oral squamous cell carcinoma mimicking osteonecrosis of the jaw: a case report
}

\author{
Vera Panzarella ${ }^{1}$, Rodolfo Mauceri ${ }^{1}$, Martina lurato Carbone ${ }^{1}$, Giacomo Oteri ${ }^{2}$, Erasmo Rubino ${ }^{2}$, Pietro Tozzo, olga di \\ fede $^{1}$, Giuseppina Campisi ${ }^{1}$ \\ 1 University of Palermo \\ 2 University of Messina
}

Funding: The author(s) received no specific funding for this work.

Potential competing interests: The author(s) declared that no potential competing interests exist.

\section{Abstract}

Osteonecrosis of the jaw (ONJ) is a well-known adverse drug reaction to bisphosphonate (BP) treatment.

ONJ diagnosis is usually based on the patient's medical/medication histories and on the clinical/radiological features of necrotic bone (frequently exposed). Furthermore, in case of mandibular involvement, patients may present pain and/or hypesthesia of the inferior alveolar nerve. There are no pathognomonic clinical and radiological signs of ONJ, and in doubtful cases, the histologic examination is recommended.

We report a case of a woman attending to our sector of Oral Medicine (AOUP "P. Giaccone" Palermo, Italy) with a mandibular bone exposure after implants loss and a medical history of BPs. Biopsy specimens demonstrated proliferation of oral squamous cell carcinoma (OSCC). Even if the role of biopsy in diagnosis ONJ is still a controversial issue, it is mandatory in case of OSCC suspicious.

Background: Osteonecrosis of the jaw $(\mathrm{ONJ})$ is an adverse effect of therapy with bisphosphonates (BP) and other antiresorptive agents. ${ }^{[1]}$ The majority of cases of ONJ observed in osteoporotic patients, treated for many years with antiresorptive drugs, with an average of 4.6 years. ${ }^{[2]}$ Clinically, it can occur as an ischemic jawbone exposed through 
fenestration of the oral mucosa or facial skin; other manifestations include pain, secondary infection, tooth loss, fistula formation and pathological fractures. ${ }^{[3]}$ The risk of $\mathrm{ONJ}$ in osteoporotic patients treated with BP remains low, compared with that of oncologic patients. However, the incidence of $\mathrm{ONJ}$ in patients in treatment with oral BPs, after dental surgical procedures, especially dental implant placements, remains uncertain. ${ }^{[4]}$

Oral squamous cell carcinoma (OSCC) accounts for $\simeq 90 \%$ of oral malignant neoplasms with a $34.70 \% 5$-year mortality rate. ${ }^{[5]}$ Current treatment of OSCC includes a combination of surgery, radiotherapy, and chemo-therapy that significantly impact patient' quality of life. ${ }^{[6][7]}$ On consequence, early diagnosis is mandatory.

Materials and Methods: We report a case of a woman who has attended our sector of Oral Medicine (AOUP "P. Giaccone" Palermo, Italy) with a local inflammation around the socket of an implant failure and slight pus excretion on the left mandible.

Results: A 62-year-old Caucasian woman presented at our sector of Oral Medicine in October 2020 with complaints of pain and swelling in his left mandible. Her medical history revealed OSCC on the left mandible on 2006, chronic druginduced pancreatitis and osteoporosis treated with alendronate from 2016 to 2018.

The patient reports mandibular rehabilitative treatment by 3 dental implants; 2 of them failed to integrate after 2 months. She had intermittent swelling and pain in the implant site and lack of soft tissue healing since the time of the fixture's explanations. Oral clinical examination showed characteristic signs of ONJ, but also an unusually high quantity of surrounding granular tissue. Due to the clinical aspects of the lesions and to medication history, a suspicion of medicationrelated osteonecrosis of the jaw was raised.

However, as the patient had a positive history for OSCC and due to the clinical appearance of the lesion, diagnostic protocol for OSCC, by magnetic resonance imaging (MRI) and incisional biopsy for histological examination, was also performed. Based on the histological and radiological findings, the definitive diagnosis of OSCC was done and appropriate oncological management has therefore been started.

Conclusions: Bisphosphonates are used in skeletal disorders, including osteoporosis, Paget's disease, bone metastases and hypercalcemia. ${ }^{[7]}$ Even though direct mechanism for $\mathrm{ONJ}$ is not fully elicited, they inhibit osteoclastic activity. ${ }^{[8]}$ The osteoclast inhibition interrupts bone resorption and weakens bone turnover remodelling and mechanical properties of the skeletal. ${ }^{[9]}$ Several local risk factors, such as periodontal disease, dental extraction, implant placement, oral infection, can increase the adverse effect of the bisphosphonates such as alendronic acid. Signs and symptoms potentially evocative of ONJ, such as bone sequestrums, suppuration, mucosa or cutaneous fistula might appear in patients with a long background of the BP administration..$^{[1][10]}$ The diagnosis of ONJ is made after clinical and radiologic investigation. ${ }^{[1]}$ Patient with ONJ are commonly treated with a combination of surgical and medical treatment. ${ }^{[4]}$

Local recurrences of OSCC occur in about $10-30 \%$ of the cases with advanced tumors, even with histopathological tumorfree surgical margins after resection. ${ }^{[11]}$ The gold standard diagnostic process for OSCC are imaging and histological confirmation. ${ }^{[12]}$

The American Society for Bone and Mineral Research recommends that the diagnosis of ONJ should specifically exclude 
other intraoral conditions, including bone tumors and metastases. ${ }^{[13]}$ Although the presence of a lesion at the site of an implant failure in a patient who has taken bisphosphonates, inevitably leads to a diagnosis of suspected osteonecrosis, biopsy and histological confirmation could be mandatory in suspicious cases.

\section{References}

1. a, b, c Salvatore L. Ruggiero, Thomas B. Dodson, John Fantasia, Reginald Goodday, Tara Aghaloo, et al. (2014). American Association of Oral and Maxillofacial Surgeons position paper on medication-related osteonecrosis of the jaw--2014 update. J Oral Maxillofac Surg. 72(10):1938-1956. doi:10.1016/j.joms.2014.04.031PubMed PMID: 25234529

2. 'Pinelopi Kleio Palaska, Vassiliki Cartsos, Athanasios I. Zavras. (2009). Bisphosphonates and Time to Osteonecrosis Development. The Oncologist. 14(11):1154-1166. doi:https://doi.org/10.1634/theoncologist.2009-0115

3. ^O. Filleul, E. Crompot, S. Saussez. (2010). Bisphosphonate-induced osteonecrosis of the jaw: a review of 2,400 patient cases. J Cancer Res Clin Oncol. 136(8):1117-1124. doi:10.1007/s00432-010-0907-7

4. ${ }^{a, b}$ Campisi G., Bedogni A., Fusco V. Raccomandazioni clinico-terapeutiche sull'osteonecrosi delle ossa mascellari (ONJ) farmaco-relata e sua prevenzione. 2020th ed. SICMF - SIPMO.

5. ^A. Capote-Moreno, P. Brabyn, M. F. Muñoz-Guerra, J. Sastre-Pérez, V. Escorial-Hernandez, et al. (2020). Oral squamous cell carcinoma: epidemiological study and risk factor assessment based on a 39-year series. International Journal of Oral and Maxillofacial Surgery. 49(12):1525-1534. doi:10.1016/.ijom.2020.03.009

6. ^ B. W. Neville, T. A. Day. (2002). Oral Cancer and Precancerous Lesions. CA: A Cancer Journal for Clinicians. 52(4):195-215. doi:10.3322/canjclin.52.4.195

7. a, bVera Panzarella, Giuseppe Pizzo, Francesco Calvino, Domenico Compilato, Giuseppe Colella, et al. (2014). Diagnostic delay in oral squamous cell carcinoma: the role of cognitive and psychological variables. Int J Oral Sci. 6(1):39-45. doi:10.1038/ijos.2013.88

8. 'Giuseppina Campisi, Stefano Fedele, Vittorio Fusco, Giuseppe Pizzo, Olga Di Fede, et al. Epidemiology, clinical manifestations, risk reduction and treatment strategies of jaw osteonecrosis in cancer patients exposed to antiresorptive agents. In: http://dx.doi.org/10.2217/fon.13.211 [Internet].: Future Medicine Ltd London, UK 3 Feb 2014[cited 1 Apr 2021]. doi:10.2217/fon.13.211

9. 'Donghyun Lee, Dong Nyoung Heo, Han-Jun Kim, Wan-Kyu Ko, Sang Jin Lee, et al. (2016). Inhibition of Osteoclast Differentiation and Bone Resorption by Bisphosphonate-conjugated Gold Nanoparticles. Sci Rep. 6(1):27336. doi:10.1038/srep27336

10. 'Anesi, Generali, Sandoni, Pozzi, Grande. (2019). From Osteoclast Differentiation to Osteonecrosis of the Jaw: Molecular and Clinical Insights. IJMS. 20(19):4925. doi:10.3390/ijms20194925

11. `Giuseppina Campisi, Rodolfo Mauceri, Francesco Bertoldo, Giordana Bettini, Matteo Biasotto, et al. (2020). Medication-Related Osteonecrosis of Jaws (MRONJ) Prevention and Diagnosis: Italian Consensus Update 2020. International Journal of Environmental Research and Public Health. 17(16):5998. doi:10.3390/ijerph17165998

12. `S. Yanamoto, S. Yamada, H. Takahashi, I. Yoshitomi, G. Kawasaki, et al. (2012). Clinicopathological risk factors for 
local recurrence in oral squamous cell carcinoma. International Journal of Oral and Maxillofacial Surgery. 41(10):11951200. doi:10.1016/j.ijom.2012.07.011

13. ^AIOM. (2019). Linee Guida TUMORI DELLA TESTA E DEL COLLO. 